\title{
Intercalibration and age of the Alder Creek sanidine ${ }^{40} \mathrm{Ar} /{ }^{39} \mathrm{Ar}$ standard
}

Elizabeth M. Niespolo ${ }^{1}$

Daniel Rutte ${ }^{1,2}$

Alan L. Deino ${ }^{2}$

Paul R. Renne ${ }^{1,2}$

${ }^{1}$ Department of Earth and Planetary Science, University of California, Berkeley, CA, 94720, USA

${ }^{2}$ Berkeley Geochronology Center, 2455 Ridge Road, Berkeley, CA, 94709, USA

\begin{abstract}
The accuracy of ${ }^{40} \mathrm{Ar} /{ }^{39} \mathrm{Ar}$ geochronology relies in large part on precise and accurate calibration of the ages and $\mathrm{K}-\mathrm{Ar}$ isotopic compositions of standards. A widely used standard for Quaternary samples, the $\sim 1.2$ Ma Alder Creek sanidine (ACs), has published ages spanning a range of $\sim 2 \%$. New measurements of ACs co-irradiated with the Fish Canyon sanidine (FCs) standard and sanidines from astronomically dated Miocene tuffs in Crete and Morocco yield results that enable both (i) a direct calibration of ACs relative to FCs, and (ii) stepwise calibrations between these two standards employing the Miocene intermediaries. Results are summarized by the parameter $R_{F C s}^{A C s}$, defined as the ratio of $\left({ }^{40} \mathrm{Ar}^{*} /{ }^{39} \mathrm{Ar}_{\mathrm{K}}\right)$ of ACs to FCs, which embodies the fundamental age relationship between these standards that is independent of systematic variables such as decay constants or absolute ages of standards. Our new measurements, executed using three mass spectrometers and various irradiation and analytical protocols, yield a weighted mean $R_{F C s}^{A C s}=0.041702 \pm 0.000014(\sigma)$. This result can be combined with previously published determinations of $R$ values for ACs relative to the Miocene tuffs and to FCs to yield a recommended interlaboratory value of $R_{F C s}^{A C s}=0.041707 \pm 0.000011$. The weighted-mean age of ACs using this interlaboratory value, based on astronomically-calibrated ages of FCs and the Miocene intermediary sanidines, is $t_{A C S}=1.1848 \pm 0.0006 \mathrm{Ma}( \pm 0.05 \%)$. Applying this result to the most precise published ${ }^{40} \mathrm{Ar} /{ }^{39} \mathrm{Ar}$ data for the MatuyamaBrunhes geomagnetic polarity reversal yields $t_{M B B}=780.1 \pm 0.8 \mathrm{ka}$. In addition, these new data for ACs support previous conclusions that U-Pb zircon ages from the Alder Creek rhyolite incorporate $\sim 13$ ka of pre-eruptive residence time.
\end{abstract}

\section{Introduction}

The ${ }^{40} \mathrm{Ar} /{ }^{39} \mathrm{Ar}$ technique is one of the most powerful dating methods available given its potential for high-precision ages and its broad applicability in a multitude of geological settings. However, the accuracy of the technique is limited by our knowledge of the true age and $\mathrm{K}-\mathrm{Ar}$ isotopic composition of standards (neutron fluence monitors) used to determine the ${ }^{40} \mathrm{Ar} * /{ }^{40} \mathrm{~K}$ ratios of unknowns through neutron activation analysis. Accuracy concerns are especially critical in the late 
Neogene, where ${ }^{40} \mathrm{Ar} /{ }^{39} \mathrm{Ar}$ ages are evaluated against comparably precise constraints from independent dating methods such as orbital tuning, ${ }^{14} \mathrm{C}$, or Useries.

Good analytical practice in ${ }^{40} \mathrm{Ar} /{ }^{39} \mathrm{Ar}$ dating dictates that the ages of standards should be as close as possible to the ages of samples being dated in order to minimize mass spectrometric biases potentially imposed by divergent ion beam intensities and their ratios. Accordingly, Turrin et al. (1994) proposed sanidine from the early Pleistocene Alder Creek rhyolite in the Napa-Sonoma volcanic province of northern California as a Quaternary (sensu lato) ${ }^{40} \mathrm{Ar} /{ }^{39} \mathrm{Ar}$ standard. This sanidine (ACs) was proposed in part because the lava was emplaced during the Cobb Mountain geomagnetic polarity event, which can in principle be dated independently by orbital tuning methods. Turrin et al. (1994) obtained an age of $1.186 \pm 0.006^{1} \mathrm{Ma}$ for this sanidine, based on an age of 27.84 Ma for the neutron fluence monitor used, sanidine from the Fish Canyon Tuff of Colorado (FCs). Renne et al. (1998) reported an age of $1.194 \pm 0.007$ Ma using 28.02 Ma for FCs, identical to the value reported by Turrin et al. (1994) when recalculated to the same age for FCs. A subsequent study (Nomade et al., 2005) of sanidine from a recollected sample yielded an age of $1.193 \pm 0.001 \mathrm{Ma}$ also using $28.02 \mathrm{Ma}$ as the age of FCs, indistinguishable from the prior two results.

More recently, several ages for ACs relative to FCs have been published by Coble et al. (2011), McDougall et al. (2012), Phillips and Matchan (2013), Rivera et al. (2013), and Jicha et al. (2016) that are distinct from the previous results as summarized by Nomade et al. (2005). Part of this inconsistency is due to different ages ascribed to the FCs standard upon which these ages are based; this is mainly because ${ }^{40} \mathrm{Ar}^{*}$ concentrations in K-Ar dated primary ('first-principals' standards calibrated through separate K and Ar measurements) standards weren't well known enough to resolve interlaboratory differences between calibrations of FCs. To isolate the effects of different calibrations versus analytical variability, we focus herein on $\mathrm{Ar}$ isotope systematics rather than computed ages. A simple metric for the age relationship between any two samples, which is independent of irradiation time or age assumed for the anchoring standard (in this case, FCs), is the $R$-value introduced by Renne et al. (1998):

$$
R_{F C s}^{A C S}=\frac{\left(e^{\lambda t_{A C S}}-1\right)}{\left(e^{\lambda t_{F C s}}-1\right)} \equiv \frac{\left({ }^{40} A r^{*} /{ }^{39} A r_{K}\right)_{A C s}}{\left({ }^{40} A r^{*} /{ }^{39} A r_{K}\right)_{F C S}}
$$

where $\lambda$ is the total ${ }^{40} \mathrm{~K}$ decay constant, ${ }^{40} A r^{*}$ is the proportion of ${ }^{40} \mathrm{Ar}$ that is radiogenic, and ${ }^{39} \mathrm{Ar}_{K}$ is the proportion of ${ }^{39} \mathrm{Ar}$ that is produced from potassium during neutron activation. $R$, which relates the age of ACs to that of FCs, may be

\footnotetext{
${ }^{1}$ All uncertainties in this paper are stated at the level of $\sigma$.
} 
determined either from corrected mass spectrometric data or from reported ages. For convenience, we define a parameter $F$ as:

$F \equiv{ }^{40} A r * /{ }^{39} A r_{K}$

such that

$R_{F C s}^{A C s}=\frac{F_{A C s}}{F_{F C s}}$

Table 1 shows a summary of $R$ for the published ACs to FCs data. The population of $R$ exhibits excess scatter about the weighted mean $(0.041787 \pm 0.000014)$, as indicated by a mean square of weighted deviates (MSWD, equivalent to the reduced chi-squared statistic) of 39.9. This indicates that the observed scatter is more than expected by the stated analytical uncertainties of the data (the data are 'overdispersed').

A contributing factor to the overdispersion may be the underestimation of error introduced in attempting to measure co-irradiated samples of ACs and FCs with ${ }^{40} \mathrm{Ar} /{ }^{39} \mathrm{Ar}$ ratios differing by a factor of $\sim 24$, which requires inevitable compromises in irradiation times and attendant differences in the behavior of ion beams, particularly of ${ }^{40} \mathrm{Ar}$ and ${ }^{39} \mathrm{Ar}$. In order to mitigate these difficulties, part of this study is designed to calibrate the ACs standard with a sample intermediate in age between ACs and FCs, allowing the $R_{F C s}^{A C s}$ value to be determined stepwise using the relationship

$R_{F C s}^{A C s}=R_{X}^{A C s} \cdot R_{F C s}^{X}$

For this purpose, sample "X" can be completely arbitrary provided it is intermediate in age between ACs and FCs and yields reproducible Ar isotope data. Ideally, it should be similar in chemical composition to ACs and FCs to minimize differences in irradiation-induced interference corrections; i.e., a sanidine is most suitable.

For this study, ACs and FCs were co-irradiated and analyzed with sanidines from an ignimbrite of the Messâdit section, Melilla basin, Morocco (Mes4) and sanidine from the A1 distal fallout tephra of the Faneromeni section at Sitia, Crete, Greece (A1). These tuffs have independent orbitally tuned ages of $6.791 \pm 0.005 \mathrm{Ma}$ and 6.943 $\pm 0.0025 \mathrm{Ma}$, respectively (Kuiper et al., 2008; Rivera et al., 2011). The Messâdit sanidine was previously analyzed by Kuiper et al. (2008) and serves in large part as basis for an orbitally tuned age of $28.201 \pm 0.023 \mathrm{Ma}$ for FCs. The A1 sanidine was used by Kuiper et al. (2004) and Rivera et al. (2011) to determine ages of 28.21 \pm 0.04 and $28.172 \pm 0.014 \mathrm{Ma}$, respectively, for FCs. Thus, in addition to serving as intermediaries in the stepwise intercalibration of ACs and FCs, independent orbitally tuned ages can be determined for ACs through Mes4 and A1. The 
calibration of ACs using astronomically tuned standards instead of primary standards minimizes the introduction of uncertainty from decay constants into the final age calculation for young Cenozoic samples (Kuiper et al, 2008). By varying grain size, irradiation time and degassing schedule (i.e., step-heating versus total fusion), and employing multi- as well as single-collector mass spectrometry, we test for the possible effects of recoil, varying ion-beam behavior, and detector intercalibration.

In addition to the stepwise intercalibration of ACs with FCs through the two intermediaries (Mes4 and A1), new direct ACs/FCs intercalibration data are presented for comparison. As shown below, these various approaches yield consistent results.

\section{Methods}

\section{Samples}

ACs (ACs-2) The Alder Creek sanidine (ACs) was obtained from the same sample (ACs-2) of the Alder Creek rhyolite analyzed by Nomade et al. (2005) from Cobb Mountain, California (N3848'6.1”, W122 $\left.{ }^{\circ} 45^{\prime} 05.4^{\prime \prime}\right)$. Phenocrystic sanidine prepared from this sample has been widely distributed to ${ }^{40} \mathrm{Ar} /{ }^{39} \mathrm{Ar}$ community worldwide. The Alder Creek rhyolite was erupted during the Cobb Mountain geomagnetic polarity subchron, whose age has been estimated by orbital tuning of marine sediments between 1.173 to $1.185 \mathrm{Ma}$ (Horng et al., 2002) and 1.190 to $1.215 \mathrm{Ma}$ (Channell et al., 2002; Shackleton et al., 1990).

Mes4 (ME00-2) Phenocrystic sanidine from sample ME00-2 (N35²1'5.48", W3 $\left.{ }^{\circ} 126.93 "\right)$ is from an ignimbrite in the Messâdit section of Kuiper et al. (2008), and it is equivalent to sample Mes4 of that study. The tuff occurs near the base of the sequence and is $\sim 5 \mathrm{~m}$ thick, distinctly thicker than any other tuff in the section. The sample analyzed on MAP 1 and a MAP215-50 instrument dubbed "Nexus" was collected by P.R.R. accompanied by K. Kuiper in 2000. A second sample (analyzed on the Noblesse) was collected in 2001 by A.L.D., accompanied by K. Kuiper.

A1 (FAN-1) Sample FAN-1 (N35¹3'19.57", E26 3'59.00") is phenocrystic sanidine from the dacitic-rhyolitic A1 tephra as identified in the Faneromeni marine sapropel cyclostratigraphic section on Crete (FAN-1; Kuiper et al., 2004; Rivera et al., 2011; Rivera et al., 2013), collected by A.L.D. accompanied by K. Kuiper in 1999.

$\underline{\text { FCs }}$ The sample of Fish Canyon Tuff was collected in 1998 (N 37³6.646', W $106^{\circ} 42.331^{\prime}$ ) by A.L.D. and C. Swisher. $264 \mathrm{~kg}$ of rock were collected and sent to M. Villeneuve at the Geological Survey of Canada for sanidine separation.

Sample preparation 
All samples were crushed, sieved, and washed in distilled water in an ultrasonic bath. Sanidine was separated using a Frantz Isodynamic magnetic separator and heavy liquids. The sanidine concentrates were cleaned with dilute hydrofluoric acid in an ultrasonic bath for 5 minutes to remove adhering groundmass, and finally handpicked to purity. Size fractions used in the various experiments are listed in Table 2.

\section{Irradiation}

Samples were irradiated in the Cd-lined, in-core CLICIT facility of the Oregon State University TRIGA reactor. Irradiation times are given in Table 2. Figure 1 shows the layout of the samples in the high-purity $\mathrm{Al}$ discs used as sample containers. In three irradiations (450PRA, 452A, and 449) the samples were mixed within individual pits and in one irradiation (424) the samples were irradiated in separate pits. Irradiation 424 contained ACs, FCs, Mes4, and A1 all in separate pits equidistant from the disc center. Planar regressions were fit to the FCs data to determine comparable $F$ values of FCs in pits containing ACs, Mes4, or A1. In irradiation 450PRA, the horizontal (within disc) fluence variations were monitored with additional ACs samples allowing assessment of maximal gradients over mixed pits and to evaluate fluence variation between separate pits.

The approximate radiogenic ${ }^{40} \mathrm{Ar}$ concentration ratio of $\sim 6$ between $\mathrm{ACs}$ and Mes4/A1 and of $\sim 4$ between Mes4/A1 and FCs allowed a reasonable compromise in irradiation time. Contrasting with an approximate ${ }^{40} \mathrm{Ar}$ concentration difference of $\sim 24$ between ACs and FCs. This highlights the advantage of the intermediate intercalibration step using $R$ values in avoiding under- or over-irradiation of one of the samples. We chose irradiation times of 3-4 $\mathrm{h}$-optimized for ACs, Mes4 and A1-for irradiations 450PRA, 452A and 449 and a more FCs-optimized irradiation time of $10 \mathrm{~h}$ for irradiation 424. In addition, in irradiation 450PRA the grain sizes of Mes4 and ACs were paired in pits with the intent to yield similarly sized ion beams of ${ }^{40} \mathrm{Ar}$ or ${ }^{39} \mathrm{Ar}$ depending on the size fraction of ACs loaded with Mes4. For equivalently sized samples, ${ }^{39} \mathrm{Ar}$ ion beams after irradiation are similar, while the combination of ACs with a smaller Mes4 fraction yields similarly sized ${ }^{40} \mathrm{Ar}$ ion beams.

Interference corrections were based on the following nucleogenic production ratios (Renne et al., 2015): $\left({ }^{40} \mathrm{Ar} /{ }^{39} \mathrm{Ar}\right)_{\mathrm{K}}=(7.30 \pm 0.92) \times 10^{-4} ;\left({ }^{37} \mathrm{Ar} /{ }^{39} \mathrm{Ar}\right)_{\mathrm{K}}=(2.24 \pm 0.16)$ $\times 10^{-4} ;\left({ }^{38} \mathrm{Ar} /{ }^{39} \mathrm{Ar}\right)_{\mathrm{K}}=(1.196 \pm 0.013) \times 10^{-2} ;\left({ }^{39} \mathrm{Ar} /{ }^{37} \mathrm{Ar}\right)_{\mathrm{Ca}}=(7.02 \pm 0.12) \times 10^{-4}$; $\left({ }^{36} \mathrm{Ar} /{ }^{37} \mathrm{Ar}\right)_{\mathrm{Ca}}=(2.702 \pm 0.004) \times 10^{-2} ;{ }^{36} \mathrm{Cl} /{ }^{38} \mathrm{Cl}=(2.628 \pm 0.002) \times 10^{2}$.

To evaluate fluence gradients over the $\mathrm{Al} \mathrm{discs,} \mathrm{the} \mathrm{two} \mathrm{distinct} \mathrm{irradiation}$ geometries used require separate treatment (Figure 1). In irradiations 449, 450PRA, and $452 \mathrm{~A}$, the various samples were irradiated in mixed pits with depths of 2-3.3 $\mathrm{mm}$ and diameters of 3.2-5.3 mm. Filling height of irradiation pits was similar, excluding influence by axial fluence gradients (i.e., variation in flux in the vertical

direction of the stacked discs). Assuming equal distribution of grains in the pits, no 
absolute correction for horizontal fluence gradients need be made when comparing average $\mathrm{F}$ values of different samples in one pit. However, given the finite spatial extent of the pits, the fluence gradient adds to the variance of the $F$ values within each pit. Rutte et al. (2015) showed that the three-dimensional variation of neutron fluence in a reactor core is expected to be the result of superposing inverse-square and exponential decay functions. However, over the small extent of the irradiation discs a planar fit is a good first order approximation with residuals of $<0.3 \%$, and mostly $<0.1 \%$. The computed gradients over individual pits are $0.04 \%(\mathrm{n}=4 ; 449 \mathrm{a})$, $0.15 \%(\mathrm{n}=9 ; 450 \mathrm{PRA})$, and $0.01 \%(\mathrm{n}=2 ; 452 \mathrm{~A})$. Thus, within-pit horizontal fluence gradients may explain up to $0.15 \%$ of the variance of $F$ values and therefore some excess dispersion in $\mathrm{F}$ values (MSWD $>1$ ). In irradiation $424, F$ values of $\mathrm{FCs}$ distributed over the disc were used to fit a planar function, representing horizontal variation in neutron fluence $(\mathrm{n}=4 ; 0.21 \% / \mathrm{cm}$, residuals $0.024 \%)$. We normalized all results of this disc, allowing comparison of $\mathrm{F}$ values of individual pits to FCs.

\section{Analytical procedures}

All analyses were conducted at the Berkeley Geochronology Center (BGC) on three mass spectrometers dubbed Nexus, MAP 1, and Noblesse.

\section{Nexus and MAP 1}

Irradiation 450PRA consisted of an eleven pit 18.54-mm aluminum disc irradiated for 4 hours. The five large pits of the disc were loaded with an even mixture of ACs and Mes4 in order to eliminate uncertainties in the neutron fluence between pits. Six additional small pits contained only ACs in order to provide additional control of neutron fluence across the disc area. Two large pits from this irradiation were analyzed on MAP1, a MAP 215-C mass spectrometer. Three large pits of mixed ACs and Mes4 as well as the six pits containing only ACs from irradiation 450PRA were analyzed on Nexus. Both MAP instruments have Nier-type ion sources and analog electron multiplier detectors.

Single grains from the large pits of mixed ACs/Mes4 underwent total laser fusion from a $\mathrm{CO}_{2}$ laser at 8 watts of power. ACs samples from small pits were measured by laser fusion analyses of 3-grain aliquots that were used only in the evaluation of fluence gradients and not in any calculations of $R$ values.

For each sample, blank, and air pipette, fifteen cycles at 8-35 integrations per cycle were measured for each Ar isotope using peak hopping by magnetic field switching on a single detector. Evolved gases were cooled to ca. -100 to $-130{ }^{\circ} \mathrm{C}$ using a cryotrap and exposed to a ca. $450{ }^{\circ} \mathrm{C}$ hot getter to remove reactive gases.

For each large pit of ACs/Mes4, a mean blank correction was determined using background isotopic measurements analyzed between each single-grain analysis. Blanks between sample measurements were stationary over time, and mean values and their standard deviations were used to correct the data for unknowns and air 
pipettes. For Nexus runs, blanks were 3-4 × 10-17, 5-15 $\times 10^{-19}$, and 7-8 $\times 10^{-19}$ moles for ${ }^{40} \mathrm{Ar},{ }^{39} \mathrm{Ar}$ and ${ }^{36} \mathrm{Ar}$, respectively. For MAP 1 runs, blanks were $1-2 \times 10^{-16}, 1-2 \times$ $10^{-18}$, and $2-3 \times 10^{-19}$ moles for ${ }^{40} \mathrm{Ar},{ }^{39} \mathrm{Ar}$ and ${ }^{36} \mathrm{Ar}$, respectively.

Mass discrimination was determined based on automated analyses of air pipettes between every five single grain analyses (plus intercalated blanks) using air pipette data based on a power law correction (Renne et al., 2009) and the atmospheric values of Lee et al. (2006); values of 1.0099 to 1.0104 and 1.0126 per Dalton were determined for Nexus and MAP 1, respectively.

\section{$\underline{\text { Noblesse }}$}

Samples analyzed on the Noblesse multicollector mass spectrometer were irradiated in three separate batches. Irradiations 449 and 452A contained pits loaded with crystals of more than one sample: 449 had four pits with both ACs and FCs standards, and 452A had four pits loaded with both of these as well as with Mes4. Irradiations 449 and 452A, like 450PRA, allow direct comparison of $F$-values for evaluation of $R$ without necessarily accounting for differences in neutron fluence.

The samples analyzed using the Noblesse multicollector instrument were degassed by incremental heating on a dedicated extraction line. Quasi-uniform heating of each sample (a single grain of sanidine) was achieved via illumination with a $\mathrm{CO}_{2}$ laser fitted with a beam-shaping lens to generate a flat energy profile of adjustable diameter, typically $2 \mathrm{~mm}$ at the target distance. Individual grains of sanidine were heated for $\sim 30$ seconds at progressively increasing power levels (0.9-8 watts) until fusion was achieved; typically 3-10 steps were employed for ACs, Mes4, and A1, and 4-13 steps for FCs. Evolved gas was exposed for several minutes to an approximately $-130^{\circ} \mathrm{C}$ cryotrap to remove $\mathrm{H}_{2} 0$, and to a GP-50 SAES getter to remove reactive gases.

Ar isotopic measurements were performed on a Noblesse 5-collector sector-magnet mass spectrometer, configured with one axial Faraday detector and four off-axis, symmetrically arrayed ETP ion counters. Prior to inlet of sample gas from the extraction line for determination of Ar isotopic composition, a 3\% aliquot of the extraction line volume was measured on the mass spectrometer within a short $(\sim 7$ s) interval. On the basis of this brief measurement, adjustments were made as necessary to the sample abundance by pumping away a calibrated fraction of the gas to evacuated ballast volumes. The goal of this procedure was to restrict the signal level of ${ }^{40} \mathrm{Ar}$ on the ion counters to less than $\sim 500 \mathrm{kcps}$ across all experiments, corresponding to a maximum ${ }^{40} \mathrm{Ar}$ of $\sim 6 \times 10^{-15}$ moles. Typically, volume splitting was unnecessary, given careful adjustment of the step-heating schedule. Subsequent to this test protocol, the remainder of the sample gas was inlet to the mass spectrometer and five Ar isotopes measured over a period of $\sim 800$ seconds. The isotopic measurement process involved simultaneous measurement of ${ }^{40} \mathrm{Ar},{ }^{37} \mathrm{Ar}$, and ${ }^{36} \mathrm{Ar}$ on separate ion counters, alternating with peak hopping to position ${ }^{38} \mathrm{Ar}$ and ${ }^{39} \mathrm{Ar}$ on the same ion counter as ${ }^{40} \mathrm{Ar}$. All signals were normalized to the ${ }^{40} \mathrm{Ar}$ ion 
counter. ${ }^{36} \mathrm{Ar}$ signal normalization was achieved through periodic measurement of the ${ }^{40} \mathrm{Ar} /{ }^{36} \mathrm{Ar}$ ratio of air argon (Lee et al., 2006) inlet from an air-reservoir pipetting system. Ar abundance from air aliquots experiments was closely matched to the unknowns, yielding $2.3 \times 10^{-15}$ moles ${ }^{40} \mathrm{Ar} .{ }^{37} \mathrm{Ar}$ and ${ }^{38} \mathrm{Ar}$ signal normalizations were achieved through periodic measurement of ${ }^{40} \mathrm{Ar}$ from a static gas sample on relevant detectors in a systematic round-robin peak-hopping procedure.

Procedural blanks, matching sample gas extractions precisely but without firing the laser, were run every four analyses and typically yielded $\sim 6 \times 10^{-17}, 4 \times 10^{-19}$, and 4 $\times 10^{-19}$ moles of ${ }^{40} \mathrm{Ar},{ }^{39} \mathrm{Ar}$, and ${ }^{36} \mathrm{Ar}$, respectively. Blanks preceding air pipette measurements were tailored specifically to mimic an actual air pipette extraction, with the exception that the inner pipette valve leading to the air reservoir was not opened. The Noblesse has sufficiently high resolution to distinguish an almost entirely hydrocarbon free shoulder at mass 36 , where the measurement for ${ }^{36} \mathrm{Ar}$ is made. For further details of the analytical procedures refer to Deino et al. (2010).

\section{Results}

$R$ values

Results in terms of inverse variance weighted mean $F$ values for each sample in each irradiation position are shown in Table 3 and Supplementary Figures 1-5. For all of the Noblesse data, which were analyzed by incremental heating, the integrated results were used for the most appropriate comparison with total fusion results from Nexus and MAP-1. Step heating data are provided as individual steps in the supplementary tables. 13 of 85 step heating results of FCs (Supplementary Figure 6) are identified as clearly rising after the first step; 5 of these had integrated ages above the mean and 8 of them had ages below the mean. However, using the same nMAD $=3$ filter as used for calculating weighted mean $F$ values, calculation of integrated ages shows no significant affect to the weighted mean age of FCs for these spectra. While some recent studies (e.g., Phillips \& Matchan, 2013; Jicha et al., 2016) have suggested that FCs is a non-ideal standard due to variations in age spectra, our data reveal little evidence to question the suitability of FCs as a standard for highprecision ${ }^{40} \mathrm{Ar} /{ }^{39} \mathrm{Ar}$ geochronology based on total gas measurements. Likewise, our step heating results for ACs produce consistent flat age spectra and a wellconstrained distribution of total gas ages (MSWD $=1.94$ ) for 71 out of 72 analyses (Supplementary Figure 7), unlike that indicated by Jicha et al. (2016). Step heating results for Mes4 and A1 can also be found as Supplementary Figures 8-9. For irradiations 449 (FCs and ACs), 450PRA (ACs and Mes4) and 452A (ACs, Mes4, and FCs), the samples were co-irradiated in the same disc position and the corresponding $R$-values for each position are shown in Table 4. For irradiation 424 (ACs, Mes4, A1, and FCs) each sample was irradiated in a separate pit and the $R$ values between each of the samples was determined by interpolation. For the determination of $F$ values for each position we used an nMAD $>3$ (normalized median absolute deviation from the median) criterion to define outliers which were then excluded from further calculations; on this basis 16 of 533 analyses were 
excluded. $R$ values were then computed from the appropriate $F$ values per Equation (3).

Determinations of $R$ values relating the various samples are presented in matrix form as shown in Table 5. The $R$ matrix provides a summary of intralaboratory weighted mean $R$ values between any two standards. The column header indicates the numerator of the $R$ value and the row header indicates the denominator. These $\mathrm{R}$ values may be used to integrate these data with previous and future data sets. Our seven new direct measurements of $R_{F C s}^{A C s}$ (weighted mean $R_{F C s}^{A C s}=0.041689 \pm$ 0.000020 ) are consistent with values obtained using Mes4 or A1 as intermediaries (weighted means $R_{F C s}^{A C s}=0.041714 \pm 0.000020$ and $0.041753 \pm 0.000080$, respectively). We take the weighted mean $\left(R_{F C s}^{A C s}=0.041702 \pm 0.000014,0.033 \%\right)$ of these three determinations as the best estimate of our intralaboratory value.

\section{Comparison with previous data}

Our direct determination of $R_{F C s}^{A C s}$ can be compared with previous results shown in Table 1 (Figure 2). Results differ significantly from pre-2006 studies (Turrin et al., 1994; Renne et al., 1998; Nomade et al., 2005), all of which were conducted at BGC. Several factors render our new data superior to those of the previous studies: (1) The spatial density of neutron fluence monitoring is much higher, including many co-irradiations in the same pit; (2) Choice of grain sizes and irradiation durations to optimize ${ }^{39} \mathrm{Ar}$ and ${ }^{40} \mathrm{Ar}$ ion beams and their ratios; (3) Use of 15 cycles of peakhopping (versus 7-10 cycles in the previous studies) on the single-collector (MAP) instruments provides more accurate constraints on the intercepts of ion beam intensity versus time, particularly for ${ }^{36} \mathrm{Ar}$; (4) More integrations per cycle were employed for ${ }^{36} \mathrm{Ar}$ and ${ }^{39} \mathrm{Ar}$ for the MAP data, further enhancing the accuracy of ion beam intercepts. Hence, pre-2006 results are excluded based on our well-informed confidence in the superiority of the new data. The results of Coble et al. (2011), McDougall et al. (2012), and Singer (2014) are relatively imprecise and compatible with a large range of values; hence these are excluded. We attempted to include the data of Jicha et al. (2016), obtained from the same preparation of ACs as ours, by computing total gas results for their incrementally-heated ACs samples. The uncertainties we calculate from their data are larger than reported. The MSWD calculated for $R_{F C S}^{A C s}$ from total gas results of Jicha et al. (2016) (computed from incremental heating data) is 31.4, much larger than the value of 6.41 determined from their single step fusion results. Our own total gas results for $R_{F C s}^{A C s}$, obtained with an identically configured mass spectrometer, are significantly less scattered with an MSWD value of 2.03. We suspect that Jicha et al. (2016) underestimated contributions from background or interference corrections resulting in spuriously high precision and resultant overdispersion of their data. For these reasons, we exclude the results of Jicha et al. (2016) from our interlaboratory compilation despite their reported $R_{F C S}^{A C S}$ being indistinguishable from our own. Our new results 
can be most appropriately be compared with Rivera et al. (2013) and Phillips and Matchan (2013). We note that the latter study used 2-3 grains of ACs for their stepheating analyses (D. Phillips, written communication, 2014), in contrast to all other data considered here which are based on analysis of single crystals. The weighted mean $R_{F C S}^{A C S}$ of these three sets of experiments is $0.041702 \pm 0.000011(0.026 \%)$.

A stepwise determination of $R_{F C s}^{A C S}$ may be calculated using Mes4 as an intermediary. Our eight independent measurements of $R_{M e s 4}^{A C s}$ are the first of which we are aware and these form a coherent data set with a weighted mean of $0.174099 \pm 0.000067$ $(0.038 \%)$. Our three new measurements of $R_{F C s}^{\text {Mes } 4}$ compare reasonably well with those of Kuiper et al. (2008), and accordingly we combine our data with the latter to yield an interlaboratory value of $0.239555 \pm 0.000066(0.027 \%)$. The corresponding stepwise value of $R_{F C s}^{A C s}=R_{M e s 4}^{A C S} \cdot R_{F C s}^{\text {Mes4 }}$ is $0.041706 \pm 0.000020(0.047 \%)$.

A second stepwise determination of $R_{F C s}^{A C s}$ is calculated using A1 as an intermediary. Our data yield a value of $0.041753 \pm 0.000080(0.191 \%)$, which may be compared with a value of $0.041753 \pm 0.000043(0.104 \%)$ determined from the data of Rivera et al. (2011) and Rivera et al. (2013). The interlaboratory weighted mean of both results gives $R_{F C S}^{A C S}=0.041753 \pm 0.000038(0.091 \%)$.

The three distinct approaches to determining $R_{F C s}^{A C S}$ using interlaboratory data as discussed above are summarized in Table 6. As shown, these result in a weighted mean $R_{F C S}^{A C S}=0.041707 \pm 0.000011(0.026 \%)$, which we recommend as an interlaboratory value.

\section{Age of ACs}

In the foregoing we have derived interlaboratory values for the intercalibration of ACs with several other standards. These values are based on analysis in three different laboratories, using five different mass spectrometers (three multicollector and two single collector) employing a variety of analytical protocols. We turn now to the issue of the age of the ACs standard.

\section{Age based on astrochronology of Mes4}

Using our results for $R_{M e s 4}^{A C s}$, the astrochronologic age of $6.791 \pm 0.005 \mathrm{Ma}$ (Kuiper et al., 2008), the ${ }^{40} \mathrm{~K}$ total decay constant of $5.463 \pm 0.107 \times 10^{-10} \mathrm{a}^{-1}$ (Min et al., 2000), Equations 5 and 6 of (Renne et al., 1998) yield

$$
t_{A C s}=\frac{1}{5.463 \times 10^{-10} a^{-1}} \ln \left[\left(e^{\left(5.463 \times 10^{-10} a^{-1}\right)\left(6.791 \times 10^{6} a\right)}-1\right)(0.174099)+1\right]
$$


$=1.1841 \pm 0.00098 \mathrm{Ma}$

Age based on astrochronology of A1

Our results for $R_{A 1}^{A C s}$ combined with the $R_{A 1}^{A C S}$ value in Table 1 of Rivera et al. (2013) $(0.170462 \pm 0.000313)$ yield a weighted mean value of $0.17055 \pm 0.00022$ $(0.127 \%)$. Substituting this value and the astrochronologic age of $6.943 \pm 0.0025 \mathrm{Ma}$ for the A1 tephra into the above Equation (5) yields an independent astronomical age $\left(t_{A C S}=1.1860 \pm 0.0016 \mathrm{Ma}\right)$ that is indistinguishable from that based on Mes4 (although less precise because $R$ was less well determined).

Ages based on intercalibration with FCs

Using our preferred interlaboratory value of $R_{F C s}^{A C s}=0.041707 \pm 0.000011$ (Table 6), we can calculate an astronomical age for ACs based on the astronomical calibration of FCs from Messâdit (Kuiper et al., 2008). This is not strictly independent of the foregoing results based on Mes4, because data from Mes4 were included in that calibration. Using the astrochronological age of $28.201 \pm 0.022$ Ma for FCs (Kuiper et al., 2008), using the uncertainty which neglects contribution from the decay constant, and applying Equation (5) with appropriate substitutions, yields $t_{A C s}=$ $1.1849 \pm 0.0009 \mathrm{Ma}$, in excellent agreement with the other two astronomicallycalibrated ages. Although these three ages are not strictly independent, we take the weighted mean $t_{A C S}=1.1848 \pm 0.0006 \mathrm{Ma}(0.052 \%)$ as the best representation of all available astronomical constraints on the age of ACs.

A fundamentally different approach uses the so-called optimization calibration of (Renne et al., 2011), which is based on an optimal fit to ${ }^{40} \mathrm{~K}$ disintegration data, $\mathrm{K}$ and Ar isotopic data for the FCs standard, and pairs of ${ }^{40} \mathrm{Ar} /{ }^{39} \mathrm{Ar}$ and ${ }^{238} \mathrm{U}-206 \mathrm{~Pb}$ data representing a partial normalization to the ${ }^{238} \mathrm{U}$ decay constant. Using our preferred interlaboratory value of $R_{F C S}^{A C S}$ as above applied to the calibration of Renne et al. (2011) yields $t_{A C S}=1.1891 \pm 0.0008 \mathrm{Ma}$, which is distinct at $95 \%$ confidence from the Mes4 and FCs based astronomically calibrated ages outlined above, but not from the less precisely determined age based on A1. However, all of these ages (Table 7) are younger than the younger mode of ${ }^{238} \mathrm{U}-{ }^{206} \mathrm{~Pb}$ zircon ages $(1.1978 \pm 0.0023 \mathrm{Ma})$ inferred by Rivera et al. (2013) for the Alder Creek rhyolite based on the likelihood of pre-eruptive residence time of zircons in silicic magmas (Simon et al., 2008).

\section{Recoil?}

The data from irradiation 450PRA, wherein two different size fractions of Mes4 were coirradiated with a single size fraction of ACs, can be used to evaluate the possibility of age bias due to differential ${ }^{39} \mathrm{Ar}$ recoil effects (Hall, 2014). The ratio of ${ }^{39} \mathrm{Ar}$ ion beams for ACs relative to Mes4 for the two positions with larger Mes4 grains is $\sim 0.68$, whereas for the three positions with smaller Mes4 grains the average ratio is $\sim 1.60$. $R$ shows no correlation with the relative ${ }^{39} \mathrm{Ar}$ ion beams, from 
which we conclude that any bias due to differential ${ }^{39} \mathrm{Ar}$ recoil effects is negligible owing to the close match in grain sizes. Hence the fractional ${ }^{39} \mathrm{Ar}$ recoil loss from each sample is comparable, and the effect on the computed $R$ values is negligible as the fraction retained is the same in the $F$ values comprising the numerator and the denominator.

\section{Application to the Matuyama-Brunhes Boundary (MBB)}

As the most recent non-excursional geomagnetic polarity reversal, the Matuyama/Brunhes boundary (MBB) is arguably the single most referenced magnetostratigraphic marker in Quaternary chronostratigraphic studies. Estimates of the age of the MBB span $>20$ ka (Singer, 2014). The most precise datum available for the age of the MBB is from the Sulmona paleolake of central Italy (Sagnotti et al., 2014), where the MBB is tightly bracketed by ${ }^{40} \mathrm{Ar} /{ }^{39} \mathrm{Ar}$ dates on sanidine from intercalated tephras, and was dated to $786.1 \pm 0.8 \mathrm{ka}$ based on the ACs age calibration of Nomade et al. (2005) and the decay constants of Steiger and Jäger (1977). This age corresponds to a value of $R_{A C s}^{M B B}=0.65830 \pm 0.00067$, which combined with our values of $R_{\text {Mes }}^{A C s}$ and $R_{A 1}^{A C s}$ yields indistinguishable ages of $779.6 \pm$ 1.0 ka and $780.8 \pm 1.3 \mathrm{ka}$ for astronomical ages based on Mes4 and A1, respectively. These two independent calibrations yield a weighted mean age for the MBB of 780.1 $\pm 0.8 \mathrm{ka}$. The optimization calibration (Renne et al., 2011) of ACs applied to the MBB yields an age of $782.8 \pm 1.0 \mathrm{ka}$, indistinguishable from either of the independent astronomical ages or their weighted mean mainly due to the relatively large uncertainty in $R_{A C s}^{M B B}$. All of these results are significantly older than the age of 773.1 \pm 0.4 ka for the MBB inferred by Channell et al. (2010) based on correlation of marine oxygen isotope records to an orbitally tuned ice-volume model. While this discrepancy may have important implications for paleoceanography and/or geomagnetic field behavior, such issues are beyond the scope of this paper.

\section{Conclusions}

Our new ${ }^{40} \mathrm{Ar} /{ }^{39} \mathrm{Ar}$ measurements of the ACs standard relative to the widely-used FCs standard, directly as well as through an intermediate step using two astronomically dated sanidines (Mes4 and A1), enable determination of $R_{F C S}^{A C S}$ that is consistent with other recent measurements of this parameter, yielding an interlaboratory value with relative uncertainty of $0.026 \%$. The resulting improvement in interlaboratory consistency is reassuring, but does not completely resolve the issue of absolute calibration as the weighted mean astronomical age $(1.1848 \pm 0.0006 \mathrm{Ma})$ differs significantly (by $0.36 \%$ ) from the optimization calibrated age $(1.1891 \pm 0.0008 \mathrm{Ma})$.

Application of the interlaboratory data to the most precise ${ }^{40} \mathrm{Ar} /{ }^{39} \mathrm{Ar}$ data for the age of the Matuyama-Brunhes boundary (Sagnotti et al., 2014) yields ages for the boundary of $780.1 \pm 0.8 \mathrm{ka}$ (weighted mean astronomical age) and $782.8 \pm 1.0 \mathrm{ka}$ 
(optimization age) which are indistinguishable at the 95\% confidence level. Both of these ages are distinctly older than that ascribed to the MBB by Channell et al. (2010).

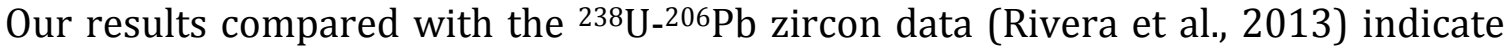
that zircons from the Alder Creek rhyolite record $13 \pm 2$ (based on the astronomical age of ACs) or $9 \pm 2 \mathrm{ka}$ (based on the optimization age of ACs) of pre-eruptive magma residence time. This conclusion agrees with that of Rivera et al. (2013), and it is noteworthy because it indicates that even the youngest mode of zircon U-Pb ages pre-dates eruption by a resolvable interval (assuming that the ${ }^{40} \mathrm{Ar} /{ }^{39} \mathrm{Ar}$ system accurately records the time of eruption). In this case the bias is small compared with some other examples (Simon et al., 2008; Wotzlaw et al., 2014), but it raises the question of what distinct petrologic event, if any, is recorded by zircon $\mathrm{U}-\mathrm{Pb}$ ages in cases wherein a well-defined and geochemically distinct youngest age mode is present. If such modes represent the integrated history of zircon crystallization (Ickert et al., 2015), then they may cluster well but still be biased by tens or hundreds of ka relative to the eruption age.

\section{Acknowledgements}

General research support is provided by the Ann and Gordon Getty Foundation. Support from the National Science Foundation EAR - 1322017 is appreciated. E.M.N. thanks U.C. Berkeley for support in the form of a Berkeley Fellowship. D.R. was supported by a DFG Research Fellowship. We thank two anonymous reviewers for insightful and constructive reviews. E.M.N. thanks Tiffany Rivera for her suggestions. The authors thank Tim Becker and Abed (Abdur-Rahim) Jaouni for laboratory and technical support. We also thank the authors of Nomade et al. (2005) for collecting the sample of ACs used in this study. 


\section{Figures}

Figure 1. Disc maps for each of the 18.54-mm aluminum discs showing samples analyzed in this study. ACs and Mes4 samples in irradiation 450PRA have the same lab ID numbers and were distinguished during analysis by ${ }^{40} \mathrm{Ar}^{*} /{ }^{39} \mathrm{Ar}_{\mathrm{K}}$.

Figure 2. Compilation of $R_{F C s}^{A C s}$ values from previous publications and this work (T94: Turrin et al. (1994); R98: Renne et al. (1998); N05: Nomade et al. (2005); C11: Coble et al. (2011); M12: McDougall et al. (2012); R13: Rivera et al. (2013); P13: Phillips \& Matchan (2013); S14: Singer et al. (2014); J16: Jicha et al. (2016)). Solid circles identify $R_{F C s}^{A C S}$ that were incorporated into the weighted mean interlaboratory $R_{F C s}^{A C s}$ represented by the shaded gray bar (see text for justification). 


\section{Supplementary Tables}

Supplementary Table 1 . Table of all ${ }^{40} \mathrm{Ar} /{ }^{39} \mathrm{Ar}$ data.

\section{Supplementary Figures}

Supplementary Figure 1. F-values of all samples included in the weighted mean Fvalue for each pit (highlighted in black above its respective column) from irradiation 450PRA. Samples displayed in light gray were excluded from the weighted mean due to $\mathrm{nMAD}>3$.

Supplementary Figure 2. F-values for samples from irradiation 452A. FCs results are plotted twice to show reduction of FCs with respect to the paired unknown displayed above it. Weighted mean F values for FCs from this irradiation reported in Table 3 are based on the reduction of FCs compared with ACs.

Supplementary Figure 3. F-values for samples from irradiation 449. Samples displayed in light gray were excluded from the weighted mean due to nMAD $>3$.

Supplementary Figure 4. F-values for samples from irradiation 424 plotted with the appropriate FCs reduction. Weighted mean F values for FCs from this irradiation reported in Table 3 are based on the reduction of FCs compared with ACs and A1.

Supplementary Figure 5. F-values for Mes4 and FCs samples from irradiation 424 plotted with the appropriate FCs reduction.

Supplementary Figure 6. a) Step heating spectra for FCs analyses on Noblesse; b) probability distribution function of integrated ages of $\mathrm{FC}$; c) probability distribution function of plateau ages of FCs yielding the same weighted mean age of FCs as the integrated ages. Figures b) and c) show results in the center plot at $2 \sigma$ uncertainty, while the weighted mean of the probability distribution function in the lowest plot is presented with $\pm 1 \sigma$ uncertainty. Outliers (in red) were omitted based on nMAD > 3 filter.

Supplementary Figure 7. a) Step heating spectra for ACs analyses on Noblesse; b) probability distribution function of integrated ages of ACs with omitted points in red (gray in grayscale); c) probability distribution function of plateau ages of ACs yielding the same weighted mean age of ACs as the integrated ages. Figures b) and c) show results in the center plot at $2 \sigma$ uncertainty, while the weighted mean of the probability distribution function in the lowest plot is presented with $\pm 1 \sigma$ uncertainty. Outliers (in red) were omitted based on nMAD $>3$ filter.

Supplementary Figure 8. Step heating spectra for Mes4 analyses on Noblesse.

Supplementary Figure 9. Step heating spectra for A1 analyses on Noblesse. 


\section{References}

Channell, J.E.T., Hodell, D.A., Singer, B.S., Xuan, C., 2010. Reconciling astrochronological and ${ }^{40} \mathrm{Ar} /{ }^{39} \mathrm{Ar}$ ages for the Matuyama-Brunhes boundary and late Matuyama Chron. Geochemistry Geophysics Geosystems 11.

Channell, J.E.T., Mazaud, A., Sullivan, P., Turner, S., Raymo, M.E., 2002. Geomagnetic excursions and paleointensities in the Matuyama Chron at Ocean Drilling Program Sites 983 and 984 (Iceland Basin). Journal of Geophysical ResearchSolid Earth 107.

Coble, M.A., Grove, M., Calvert, A.T., 2011. Calibration of Nu-Instruments Noblesse multicollector mass spectrometers for argon isotopic measurements using a newly developed reference gas. Chemical Geology 290, 75-87.

Deino, A.L., Scott, G.R., Saylor, B., Alene, M., Angelini, J.D., Haile-Selassie, Y., 2010. ${ }^{40} \mathrm{Ar} /{ }^{39} \mathrm{Ar}$ dating, paleomagnetism, and tephrochemistry of Pliocene strata of the hominid-bearing Woranso-Mille area, west-central Afar Rift, Ethiopia. Journal of Human Evolution 58, 111-126.

Hall, C.M., 2014. Direct measurement of recoil effects on ${ }^{40} \mathrm{Ar} /{ }^{39} \mathrm{Ar}$ standards. Advances in ${ }^{40} \mathrm{Ar} /{ }^{39} \mathrm{Ar}$ Dating: From Archaeology to Planetary Sciences 378, 53-62.

Horng, C.S., Lee, M.Y., Palike, H., Wei, K.Y., Liang, W.T., 2002. Astronomically calibrated ages for geomagnetic reversals within the Matuyama chron. Earth Planets and Space 54, 679-690.

Ickert, R.B., Mundil, R., Magee, C.W., Jr., Mulcahy, S.R., 2015, The U-Th-Pb systematics of zircon from the Bishop Tuff: A case study in challenges to high-precision $\mathrm{Pb} / \mathrm{U}$ geochronology at the millenial scale: Geochimica et Cosmochimica Acta 168, 88-110.

Jicha, B.R., Singer, B.S., Sobol, P., 2016. Re-evaluation of the ages of ${ }^{40} \mathrm{Ar} /{ }^{39} \mathrm{Ar}$ sanidine standards and supereruptions in the western US using a Noblesse multi-collector mass spectrometer. Chemical Geology 431, 54-66.

Kuiper, K.F., Deino, A., Hilgen, F.J., Krijgsman, W., Renne, P.R., Wijbrans, J.R., 2008. Synchronizing rock clocks of Earth history. Science 320, 500-504.

Kuiper, K.F., Hilgen, F.J., Steenbrink, J., Wijbrans, J.R., 2004. ${ }^{40} \mathrm{Ar} /{ }^{39} \mathrm{Ar}$ ages of tephras intercalated in astronomically tuned Neogene sedimentary sequences in the eastern Mediterranean. Earth and Planetary Science Letters 222, 583-597.

Lee, J.Y., Marti, K., Severinghaus, J.P., Kawamura, K., Yoo, H.S., Lee, J.B., Kim, J.S., 2006. A redetermination of the isotopic abundances of atmospheric Ar. Geochimica et Cosmochimica Acta 70, 4507-4512.

McDougall, I., Brown, F.H., Vasconcelos, P.M., Cohen, B.E., Thiede, D.S., Buchanan, M.J., 2012. New single crystal ${ }^{40} \mathrm{Ar} /{ }^{39} \mathrm{Ar}$ ages improve time scale for deposition of the Omo Group, Omo-Turkana Basin, East Africa. Journal of the Geological Society 169, 213-226.

Min, K.W., Mundil, R., Renne, P.R., Ludwig, K.R., 2000. A test for systematic errors in ${ }^{40} \mathrm{Ar} /{ }^{39} \mathrm{Ar}$ geochronology through comparison with $\mathrm{U} / \mathrm{Pb}$ analysis of a 1.1-Ga rhyolite. Geochimica et Cosmochimica Acta 64, 73-98. 
Nomade, S., Renne, P.R., Vogel, N., Deino, A.L., Sharp, W.D., Becker, T.A., Jaouni, A.R., Mundil, R., 2005. Alder Creek sanidine (ACs-2): A Quaternary ${ }^{40} \mathrm{Ar} /{ }^{39} \mathrm{Ar}$ dating standard tied to the Cobb Mountain geomagnetic event. Chemical Geology 218, 315-338.

Phillips, D., Matchan, E.L., 2013. Ultra-high precision ${ }^{40} \mathrm{Ar} /{ }^{39} \mathrm{Ar}$ ages for Fish Canyon Tuff and Alder Creek Rhyolite sanidine: New dating standards required? Geochimica et Cosmochimica Acta 121, 229-239.

Renne, P.R., Balco, G., Ludwig, K.R., Mundil, R., Min, K., 2011. Response to the comment by W.H. Schwarz et al. on "Joint determination of ${ }^{40} \mathrm{~K}$ decay constants and ${ }^{40} \mathrm{Ar} * /{ }^{40} \mathrm{~K}$ for the Fish Canyon sanidine standard, and improved accuracy for ${ }^{40} \mathrm{Ar} /{ }^{39} \mathrm{Ar}$ geochronology" by PR Renne et al. (2010). Geochimica et Cosmochimica Acta 75, 5097-5100.

Renne, P.R., Cassata, W.S., Morgan, L.E., 2009. The isotopic composition of atmospheric argon and ${ }^{40} \mathrm{Ar} /{ }^{39} \mathrm{Ar}$ geochronology: Time for a change? Quaternary Geochronology 4, 288-298.

Renne, P.R., Swisher, C.C., Deino, A.L., Karner, D.B., Owens, T.L., DePaolo, D.J., 1998. Intercalibration of standards, absolute ages and uncertainties in ${ }^{40} \mathrm{Ar} /{ }^{39} \mathrm{Ar}$ dating. Chemical Geology 145, 117-152.

Renne, P.R., Sprain, C.J., Richards, M.A., Self, S., Vanderkluysen, L., Pande, K., 2015. State shift in Deccan volcanism at the Cretaceous-Paleogene boundary, possibly induced by impact. Science 350, 76-78.

Rivera, T.A., Storey, M., Schmitz, M.D., Crowley, J.L., 2013. Age intercalibration of ${ }^{40} \mathrm{Ar} /{ }^{39} \mathrm{Ar}$ sanidine and chemically distinct $\mathrm{U} / \mathrm{Pb}$ zircon populations from the Alder Creek Rhyolite Quaternary geochronology standard. Chemical Geology 345, 87-98.

Rivera, T.A., Storey, M., Zeeden, C., Hilgen, F.J., Kuiper, K., 2011. A refined astronomically calibrated ${ }^{40} \mathrm{Ar} /{ }^{39} \mathrm{Ar}$ age for Fish Canyon sanidine. Earth and Planetary Science Letters 311, 420-426.

Rutte, D., Pfander, J.A., Koleska, M., Jonckheere, R., Unterricker, S., 2015. Radial fastneutron fluence gradients during rotating ${ }^{40} \mathrm{Ar} /{ }^{39} \mathrm{Ar}$ sample irradiation recorded with metallic fluence monitors and geological age standards. Geochemistry Geophysics Geosystems 16, 336-345.

Sagnotti, L., Scardia, G., Giaccio, B., Liddicoat, J.C., Nomade, S., Renne, P.R., Sprain, C.J., 2014. Extremely rapid directional change during Matuyama-Brunhes geomagnetic polarity reversal. Geophysical Journal International 199, 11101124.

Shackleton, N.J., Berger, A., Peltier, W.R., 1990. An alternative astronomical calibration of the lower Pleistocene timescale based on ODP site 677. Transactions of the Royal Society of Edinburgh-Earth Sciences 81, 251-261.

Simon, J.I., Renne, P.R., Mundil, R., 2008. Implications of pre-eruptive magmatic histories of zircons for U-Pb geochronology of silicic extrusions. Earth and Planetary Science Letters 266, 182-194.

Singer, B.S., 2014. A Quaternary geomagnetic instability time scale. Quaternary Geochronology 21, 29-52. 
Steiger, R.H., Jager, E., 1977. Subcomission on geochronology: Convention on use of decay constants in geochronology and cosmochronology. Earth and Planetary Science Letters 36, 359-362.

Turrin, B.D., Donnellynolan, J.M., Hearn, B.C., $1994 .{ }^{40} \mathrm{Ar} /{ }^{39} \mathrm{Ar}$ ages from the rhyolite of Alder Creek, California: Age of the Cobb Mountain Normal-Polarity Subchron revisited. Geology 22, 251-254.

Wotzlaw, J.-F., Husing, S.K., Hilgen, F.J., Schaltegger, U., 2014. High-precision zircon $\mathrm{U}-\mathrm{Pb}$ geochronology of astronomically dated volcanic ash beds from the Mediterranean Miocene. Earth and Planetary Science Letters 407, 19-34. 


\begin{tabular}{lccc}
\hline \multicolumn{1}{c}{ Publication } & R & \pm (abs.) & $\pm(\mathbf{\%})$ \\
\hline Turrin et al. (1994) & 0.04229 & 0.00021 & $0.50 \%$ \\
Renne et al. (1998) & 0.04229 & 0.00006 & $0.14 \%$ \\
Nomade et al. (2005) & 0.04230 & 0.00006 & $0.14 \%$ \\
Coble et al. (2011) & 0.04180 & 0.00021 & $0.50 \%$ \\
McDougall et al. (2012) & 0.04189 & 0.0006 & $1.43 \%$ \\
Rivera et al. (2013) & 0.04175 & 0.00003 & $0.07 \%$ \\
Phillips \& Matchan (2013) & 0.04169 & 0.00002 & $0.04 \%$ \\
Singer (2014) & 0.04185 & 0.00011 & $0.27 \%$ \\
Jicha et al. (2016) & 0.04176 & 0.00004 & $0.09 \%$ \\
\hline
\end{tabular}

Table 1. Summary of $R_{F C S}^{A C s}$ from published data reflect excess scatter yielding an imprecise and potentially inaccurate calibration of ACs. 


\begin{tabular}{|c|c|c|c|c|c|c|}
\hline \multicolumn{3}{|c|}{ Irradiation } & \multicolumn{4}{|c|}{ Size fractions $(\mu \mathrm{m})$} \\
\hline Number & $\begin{array}{l}\text { Time } \\
\text { (h) }\end{array}$ & Layout & ACs & Mes4 & A1 & $\mathrm{FCs}$ \\
\hline 450PRA & $4 \mathrm{~h}$ & mixed pits & $841-1410$ & $595-841,841-1410$ & - & - \\
\hline 424 & $10 \mathrm{~h}$ & separate pits & $600-700$ & $500-600$ & $500-600$ & $300-350$ \\
\hline $452 \mathrm{~A}$ & $3 \mathrm{~h}$ & mixed pits & $700-840$ & $500-600$ & - & $400-500$ \\
\hline 449 & $3 \mathrm{~h}$ & mixed pits & $700-840$ & - & - & $350-400$ \\
\hline
\end{tabular}

Table 2. Size fractions and irradiation times for each disk. See Figure 1 for disk configurations and sample arrangements. 


\begin{tabular}{|c|c|c|c|c|c|c|c|}
\hline Irradiation & Pit \# & $\begin{array}{c}\text { Lab } \\
\text { ID }\end{array}$ & Sample & $\begin{array}{c}\text { Wtd. Mean } \\
\text { F }\end{array}$ & \pm (abs.) & $\pm(\%)$ & $\begin{array}{c}\text { Number of } \\
\text { grains included } \\
\text { (excluded) }^{\mathrm{a}}\end{array}$ \\
\hline \multirow[t]{10}{*}{ 450PRA } & \multirow{2}{*}{6} & \multirow{2}{*}{37049} & ACs & 0.61929 & 0.00045 & 0.074 & $28(1)$ \\
\hline & & & Mes4 & 3.5532 & 0.0011 & 0.032 & $30(2)$ \\
\hline & \multirow{2}{*}{7} & \multirow{2}{*}{37050} & $\mathrm{ACs}$ & 0.61972 & 0.00043 & 0.069 & $22(2)$ \\
\hline & & & Mes4 & 3.5653 & 0.0008 & 0.022 & $23(0)$ \\
\hline & \multirow{2}{*}{8} & \multirow{2}{*}{37051} & $\mathrm{ACs}$ & 0.62072 & 0.00052 & 0.084 & $30(0)$ \\
\hline & & & Mes4 & 3.5622 & 0.0010 & 0.029 & $32(1)$ \\
\hline & \multirow{2}{*}{9} & \multirow{2}{*}{37052} & $\mathrm{ACs}$ & 0.62315 & 0.00074 & 0.12 & $30(0)$ \\
\hline & & & Mes4 & 3.5798 & 0.0016 & 0.044 & $26(3)$ \\
\hline & \multirow{2}{*}{10} & \multirow{2}{*}{37053} & $\mathrm{ACs}$ & 0.62453 & 0.00073 & 0.12 & $25(0)$ \\
\hline & & & Mes4 & 3.5882 & 0.0012 & 0.033 & $26(0)$ \\
\hline \multirow[t]{11}{*}{424} & 13 & 26441 & $\mathrm{ACs}$ & 0.25263 & 0.00029 & 0.11 & $14(1)$ \\
\hline & 3 & 26430 & Mes4 & 1.45013 & 0.00072 & 0.050 & $12(1)$ \\
\hline & 7 & 26434 & Mes4 & 1.44807 & 0.00064 & 0.044 & $13(2)$ \\
\hline & 11 & 26438 & Mes4 & 1.45128 & 0.00063 & 0.043 & $14(0)$ \\
\hline & 15 & 26442 & Mes4 & 1.45161 & 0.00068 & 0.047 & $14(0)$ \\
\hline & 6 & 26433 & A1 & 1.47732 & 0.00082 & 0.056 & $15(0)$ \\
\hline & 16 & 26443 & A1 & 1.48139 & 0.00094 & 0.063 & $17(0)$ \\
\hline & 1 & 26428 & $\mathrm{FCs}$ & 6.0471 & 0.0024 & 0.039 & $11(0)$ \\
\hline & 5 & 26432 & FCs & 6.0443 & 0.0026 & 0.043 & $10(0)$ \\
\hline & 9 & 26436 & FCs & 6.0433 & 0.0021 & 0.035 & $10(0)$ \\
\hline & 13 & 26440 & FCs & 6.0515 & 0.0025 & 0.041 & $10(0)$ \\
\hline \multirow[t]{8}{*}{449} & \multirow{2}{*}{1} & 27023 & $\mathrm{ACs}$ & 0.8583 & 0.0010 & 0.11 & $7(1)$ \\
\hline & & 27010 & $\mathrm{FCs}$ & 20.646 & 0.012 & 0.058 & $6(0)$ \\
\hline & \multirow{2}{*}{4} & 27026 & ACs & 0.86083 & 0.00085 & 0.10 & $11(0)$ \\
\hline & & 27013 & $\mathrm{FCs}$ & 20.6600 & 0.0092 & 0.044 & $7(0)$ \\
\hline & \multirow{2}{*}{7} & 27028 & $\mathrm{ACs}$ & 0.86173 & 0.00091 & 0.11 & $10(0)$ \\
\hline & & 27016 & FCs & 20.6288 & 0.0087 & 0.042 & $7(0)$ \\
\hline & \multirow{2}{*}{10} & 27030 & $\mathrm{ACs}$ & 0.85600 & 0.00079 & 0.09 & $9(1)$ \\
\hline & & 27019 & $\mathrm{FCs}$ & 20.6289 & 0.0088 & 0.043 & $6(0)$ \\
\hline \multirow[t]{6}{*}{$452 \mathrm{~A}$} & \multirow{4}{*}{1} & 27127 & $\mathrm{ACs}$ & 0.83806 & 0.00088 & 0.10 & $11(1)$ \\
\hline & & 27126 & Mes4 & 4.8128 & 0.0022 & 0.046 & $10(0)$ \\
\hline & & 27114 & FCs & 20.0787 & 0.0069 & 0.034 & $9(0)$ \\
\hline & & 27130 & $\mathrm{ACs}$ & 0.83671 & 0.00088 & 0.10 & $9(0)$ \\
\hline & \multirow[t]{2}{*}{4} & 27129 & Mes4 & 4.8072 & 0.0023 & 0.048 & $10(0)$ \\
\hline & & 27117 & FCs & 20.0738 & 0.0064 & 0.032 & $9(0)$ \\
\hline
\end{tabular}

${ }^{a}$ Filtered with a normalized median absolute deviation (nMAD) threshold of 3

Table 3. Weighted mean F-values of individual pits from all irradiations in this study. 


\begin{tabular}{|c|c|c|c|c|}
\hline Lab ID & $R_{M e s 4}^{A C s}$ & \pm (abs.) & $\pm(\%)$ & Irradiation \\
\hline 37049 & 0.174292 & 0.000140 & 0.080372 & 450PRA \\
\hline 37050 & 0.173821 & 0.000126 & 0.072562 & 450PRA \\
\hline 37051 & 0.174253 & 0.000154 & 0.088388 & 450PRA \\
\hline 37052 & 0.174074 & 0.000220 & 0.126425 & 450PRA \\
\hline 37053 & 0.174051 & 0.000213 & 0.122101 & 450PRA \\
\hline $27127 / 27126$ & 0.174232 & 0.000319 & 0.183017 & $452 \mathrm{~A}$ \\
\hline $27130 / 27129$ & 0.174152 & 0.000430 & 0.247127 & $452 \mathrm{~A}$ \\
\hline$\left(R_{F C s}^{A C s}\right) /\left(R_{F C s}^{M e s 4}\right)$ & 0.174193 & 0.000289 & 0.165648 & 424 \\
\hline Lab ID & $R_{F C s}^{M e s 4}$ & \pm (abs.) & $\pm(\%)$ & Irradiation \\
\hline $27126 / 27114$ & 0.239551 & 0.000177 & 0.073805 & $452 \mathrm{~A}$ \\
\hline $27129 / 27117$ & 0.239339 & 0.000159 & 0.066558 & $452 \mathrm{~A}$ \\
\hline Irr. 424 & 0.239694 & 0.000089 & 0.037166 & 424 \\
\hline Lab ID & $R_{F C s}^{A C s}$ & \pm (abs.) & $\pm(\%)$ & Irradiation \\
\hline $27127 / 27114$ & 0.041737 & 0.000070 & 0.167476 & $452 \mathrm{~A}$ \\
\hline $27130 / 27117$ & 0.041682 & 0.000099 & 0.237995 & $452 \mathrm{~A}$ \\
\hline $27023 / 27010$ & 0.041546 & 0.000050 & 0.121312 & 449 \\
\hline $27026 / 27013$ & 0.041671 & 0.000045 & 0.107029 & 449 \\
\hline $27028 / 27016$ & 0.041773 & 0.000044 & 0.104135 & 449 \\
\hline $27030 / 27019$ & 0.041672 & 0.000042 & 0.100786 & 449 \\
\hline 26441 & 0.041753 & 0.000067 & 0.161425 & 424 \\
\hline Calculated & 0.041655 & 0.000035 & 0.083354 & $\begin{array}{c}R_{M e s 4}^{A C s}(450 \mathrm{PRA}) \times \\
R_{F C s}^{M e s 4}(\text { Kuiper et al., } 2008)\end{array}$ \\
\hline
\end{tabular}

Irradiation 424 individual pit $\mathrm{R}$ values vs. FCs

\begin{tabular}{ccccc} 
Lab ID & $R_{F C s}^{U n k}$ & $\pm(\mathbf{a b s})$ & $\pm(\mathbf{\%})$ & Sample ID “Unk” \\
\hline 26430 & 0.239658 & 0.000214 & 0.089085 & Mes4 \\
26433 & 0.244508 & 0.000251 & 0.102614 & A1 \\
26434 & 0.239524 & 0.000145 & 0.060662 & Mes4 \\
26438 & 0.239932 & 0.000194 & 0.080648 & Mes4 \\
26441 & 0.041753 & 0.000067 & 0.160948 & ACs \\
26442 & 0.239779 & 0.000183 & 0.076154 & Mes4 \\
26443 & 0.244866 & 0.000252 & 0.102954 & A1 \\
\hline
\end{tabular}

Table 4. $R$ values from co-irradiated samples in irradiations 450PRA, 452A, and 449, and calculated $R$ values from irradiation 424 as described in the text. Under $R_{F C s}^{M e s 4}$ the four pits of Mes4 in irradiation 424 are given as a weighted mean $R$ value (details in text). 


\begin{tabular}{|c|c|c|c|c|}
\hline$R_{\text {Row }}^{\text {Column }}$ & ACs & Mes4 & A1 & FCs \\
\hline ACs & 1 & $\begin{array}{c}5.743859 \\
\pm 0.002195 \\
( \pm 0.038 \%)\end{array}$ & $\begin{array}{c}5.860311 \\
\pm 0.010375 \\
( \pm 0.177 \%)\end{array}$ & $\begin{array}{l}23.993239 \\
\pm 0.009950 \\
( \pm 0.041 \%)\end{array}$ \\
\hline Mes4 & $\begin{array}{c}0.174099 \\
\pm 0.000067 \\
( \pm 0.038 \%)\end{array}$ & 1 & $\begin{array}{c}1.020825 \\
\pm 0.000833 \\
( \pm 0.082 \%)\end{array}$ & $\begin{array}{c}4.173618 \\
\pm 0.001240 \\
( \pm 0.030 \%)\end{array}$ \\
\hline A1 & $\begin{array}{c}0.170639 \\
\pm 0.000302 \\
( \pm 0.177 \%)\end{array}$ & $\begin{array}{c}0.979600 \\
\pm 0.000800 \\
( \pm 0.082 \%)\end{array}$ & 1 & $\begin{array}{c}4.086868 \\
\pm 0.002970 \\
( \pm 0.073 \%)\end{array}$ \\
\hline FCs & $\begin{array}{c}0.041678 \\
\pm 0.000017 \\
( \pm 0.041 \%)\end{array}$ & $\begin{array}{c}0.239600 \\
\pm 0.000071 \\
( \pm 0.030 \%)\end{array}$ & $\begin{array}{c}0.244686 \\
\pm 0.000178 \\
( \pm 0.073 \%)\end{array}$ & 1 \\
\hline
\end{tabular}

Table 5. Matrix of intralaboratory weighted mean $\mathrm{R}$ values for all iterations of the four samples evaluated. 


\begin{tabular}{cccc}
\hline Method & $R_{F C s}^{A C S}$ & $\pm \sigma$ & $\pm \sigma(\%)$ \\
\hline Direct & 0.041702 & 0.000011 & $0.03 \%$ \\
Via Mes4 & 0.041706 & 0.000020 & $0.05 \%$ \\
Via A1 & 0.041753 & 0.000038 & $0.09 \%$ \\
Weighted Mean & $\mathbf{0 . 0 4 1 7 0 7}$ & $\mathbf{0 . 0 0 0 0 1 1}$ & $\mathbf{0 . 0 3 \%}$ \\
\hline
\end{tabular}

Table 6. Interlaboratory values of $R_{F C s}^{A C s}$ obtained by different methods. 


\begin{tabular}{cccc}
\hline Method & $\begin{array}{c}\text { Age of ACs } \\
(\mathrm{Ma})\end{array}$ & $\pm \sigma(\mathrm{Ma})$ & $\pm \sigma(\%)$ \\
\hline Via FCs* & 1.1849 & 0.0009 & $0.08 \%$ \\
Via Mes4 & 1.1841 & 0.0010 & $0.08 \%$ \\
Via A1 & 1.1860 & 0.0016 & $0.13 \%$ \\
\hline Astronomical & $\mathbf{1 . 1 8 4 8}$ & $\mathbf{0 . 0 0 0 6}$ & $\mathbf{0 . 0 5 \%}$ \\
Optimization & $\mathbf{1 . 1 8 9 1}$ & $\mathbf{0 . 0 0 0 8}$ & $\mathbf{0 . 0 7 \%}$ \\
*Based on all Messâdit section tuning data of Kuiper et al., 2008 \\
\hline
\end{tabular}

Table 7. Summary of ages for ACs. Astronomical result shows the weighted mean of three ages via FCs, Mes4, and A1 calibrations above. Optimization result shows the age of ACs using the weighted mean interlaboratory $R_{F C s}^{A C s}$ in the optimization calibration (Renne et al., 2011). 

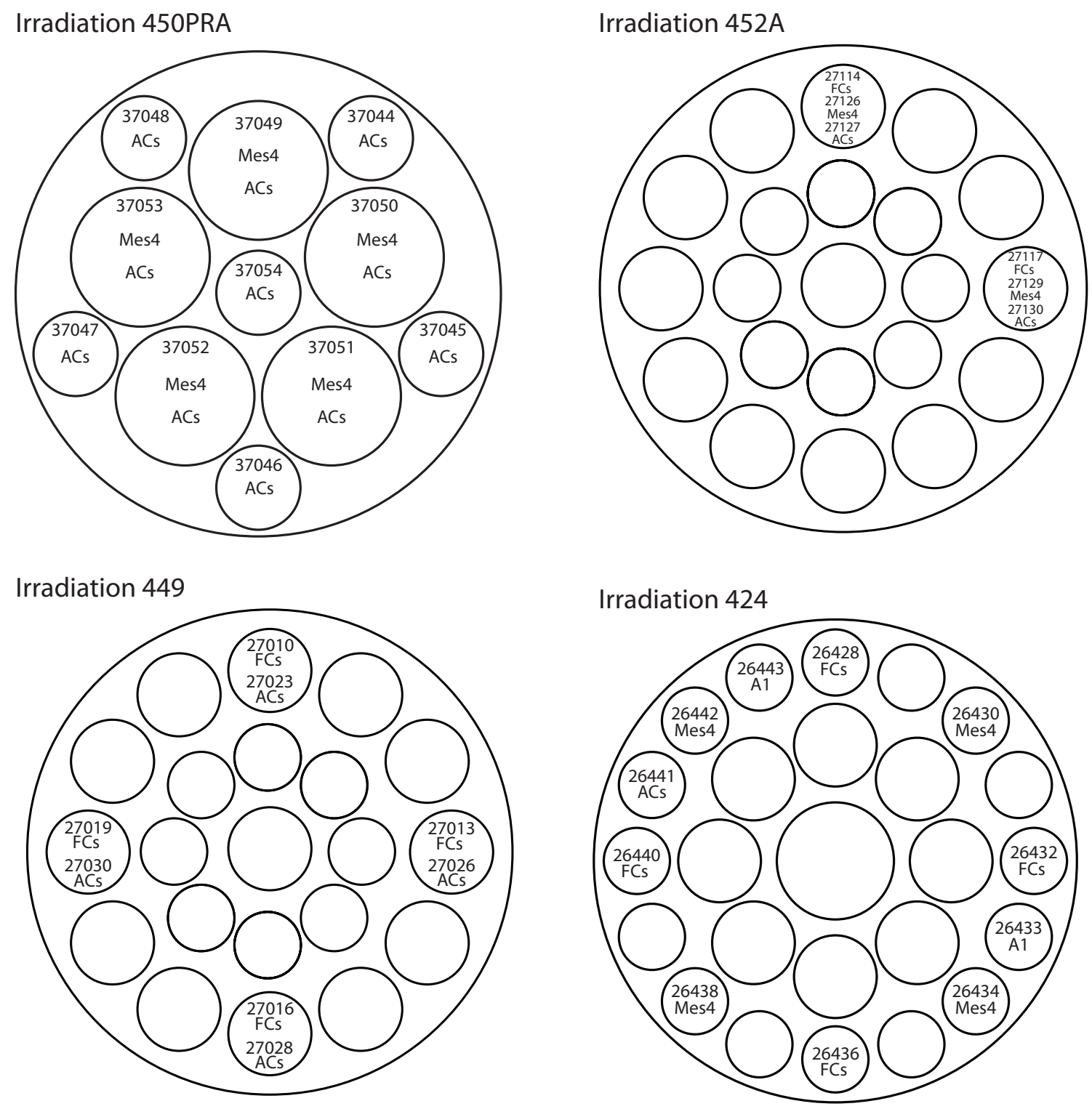

Figure 1 Niespolo et al. 


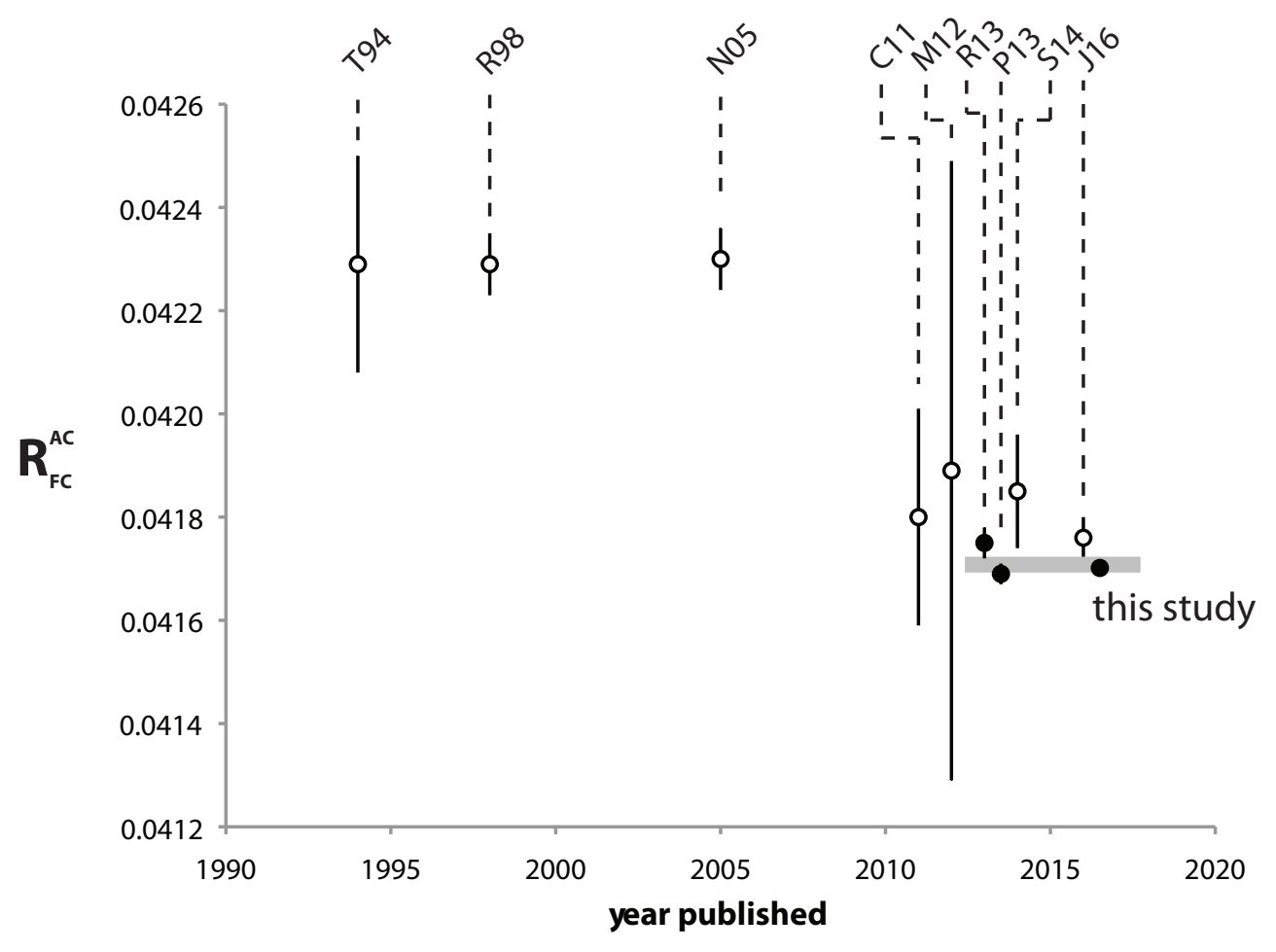

Figure 2 Niespolo et al. 